УДК: 616.314-77

DOI

\title{
ВЫСОКАЯ ТОЧНОСТЬ КОНСТРУКЦИЙ ПРИ ПРИМЕНЕНИИ 3D-ПЕЧАТИ В ИМПЛАНТОЛОГИИ (ОБЗОР ЛИТЕРАТУРЫ)
}

\section{HIGH ACCURACY OF DESIGNS WHEN USING 3D PRINTING IN IMPLANTOLOGY (REVIEW OF LITERATURE)}

\author{
В.А. Иванова, В.В. Борисов, В.В. Платонова, С.Д. Даньшина \\ V.A. Ivanova, V.V. Borisov, V.V. Platonova, S.D. Danshina
}

Первый Московский государственный медицинский университет имени И.М. Сеченова, Россия, 119991, г. Москва, ул. Трубецкая, д. 8, стр. 2

Moscow State Medical University (Sechenov University), 8-2 Trubetskaya St, Moscow, 119991, Russia

E-mail: karapeta@narod.ru

\begin{abstract}
Аннотация
3D-печать приветствовалась как технология, которая изменит развитие стоматологии. 3D-печать становится предметом большого интереса в челюстно-лицевой хирургии, ортопедии, эндодонтии, ортодонтии и пародонтологии. Эта технология играет большую роль в лечении стоматологических заболеваний, и с развитием технологий 3D-визуализации и моделирования, таких как конуснолучевая компьютерная томография и интраоральное сканирование, а также с относительно долгой историей использования технологий $\mathrm{CAD} / \mathrm{CAM}$ в стоматологии, она будет приобретать все большее значение. Использование 3D-печати включает в себя производство фрезерованных направляющих для зубных имплантатов, производство физических моделей для ортопедической стоматологии, ортодонтии и хирургии, производство зубных, краниомаксиллофациальных и ортопедических имплантатов, а также изготовление шаблонов и каркасов для имплантации и реставрации зубов. Трехмерное производство обладает большим количеством преимуществ: улучшение качества лечения, развитие персонализированной медицины, высокая скорость предоставления услуги, что делает 3D-печать неотделимой частью будущей стоматологии. В данной статье рассматривается точность конструкции при использовании 3D-печати. Также обсуждается зависимость точности моделей, изготовленных с помощью трехмерной печати, от ряда факторов, таких как метод 3D-печати, настройки 3D-принтера, используемая программа печати.
\end{abstract}

\begin{abstract}
3D printing was hailed as a technology that would change the development of dentistry. 3D printing is becoming a subject of great interest in maxillofacial surgery, prosthodontics, endodontics, orthodontics and periodontics. This technology plays an important role in the treatment of dental diseases, and with the development of 3D imaging and modeling technologies such as cone-beam computed tomography and intraoral scanning, as well as a relatively long history of using CAD/CAM technologies in dentistry, it will become increasingly important. The use of 3D printing includes the production of milled guides for dental implants, the production of physical models for orthopedic dentistry, orthodontics and surgery, the production of dental, craniomaxillofacial and orthopedic implants, as well as the fabrication of copings and frameworks for implant and dental restorations. Three-dimensional production has many advantages: improving the quality of treatment, developing personalized medicine and providing high-speed services, which make 3D printing an integral part of the future of dentistry. This article discusses the accuracy of the design when using 3D printing. We also discuss the dependence of the accuracy of models, made by using three-dimensional printing on a number of factors, such as the 3D printing method, the 3D printer settings and the printing program.
\end{abstract}


Ключевые слова: 3D-печать, стоматологическая модель, 3D-принтер, точность, аддитивные технологии.

Keywords: 3D printing, dental model, 3D printer, precision, additive manufacturing.

Медицинские технологии визуализации включают в себя исследования, начиная с рентгеновской радиологии и заканчивая более продвинутыми и усовершенствованными методами медицинской визуализации, такими как компьютерная томография (КТ), магнитно-резонансная томография (МРТ) и лазерная оцифровка. Эти новые технологии могут обеспечить детальные трехмерные изображения анатомии области интереса и, следовательно, ценные данные для диагностического и терапевтического применения [Liu, Leu, and Schmitt, 2006; Chan, Misch, and Wang, 2010; Shah and Chong, 2018].

Трехмерная (3D) печать - это новейшая технологическая разработка, которая может сыграть значительную роль в диагностике и лечении статмологических заболеваний [Lee et al., 2015; Shah and Chong, 2018; Oberoi et al., 2018; Tahayeri et al., 2018; Wang, Chen, and Lin, 2019]. Данный метод имеет ряд преимуществ по сравнению с обычной фрезеровкой. 3D-печать позволяет формировать объекты с любой геометрической сложностью, из различных видов соединений, композитов или материалов.

Аддитивные системы изготовления сводят построение сложных объектов к управляемому, простому и относительно быстрому процессу. Эти свойства привели к их широкому применению [Донских, 2017; Лазаренко и др., 2018]. Кроме того, 3D-печать обладает огромным потенциалом для улучшения гигиены полости рта, что подтверждается в различных исследованиях и клиническом лечении [Oberoi et al., 2018]. В последнее время для стоматологического реконструктивного лечения пациентов с челюстно-лицевыми деформациями стали применяться технологии трехмерной печати [Sinn, Cillo, and Miles, 2006; Dawood, Tanner, and Hutchison, 2013; Louvrier et al., 2017]. Распространенное использование 3D-принтеров приводит к переходу от более традиционного клинического процесса к почти исключительно цифровому формату [Kasparova et al., 2013; Визер, Елали, 2017; Brown et al., 2018; Чевычелова, Зубкова, 2019].

Визуализация результатов лечения делает его перспективным клиническим инструментом [Moser, Santander, and Quast, 2018]. Его повышенное использование в значительной степени объясняется тем, что он может обеспечить индивидуальный подход в течение короткого периода времени, что соответствует цели индивидуализированной медицины, где каждый пациент требует конкретного, индивидуального, терапевтического подхода [Dodziuk, 2016; Коровкина, 2018]. Поскольку одной из целей современной медицины является внедрение персонализированной помощи, технология 3D-печати должна применяться для поддержки таких методов, поскольку она может обеспечить конкретный подход для каждого пациента за короткий период времени с минимальными затратами и с сохранением высокого качества обслуживания [Дьяченко и др., 2015; Choi and Kim, 2015].

Как мы видим, движущая сила прогресса в области 3D-печати для медицины и стоматологии заключается в возможности индивидуализации продукции, экономии на мелкомасштабных производствах, упрощении обмена и обработки данных изображений пациентов и модернизации образования [Oberoi et al., 2018]. По мере развития знаний, аспирантские программы должны рассмотреть возможность внедрения 3D-печати в свои учебные программы [Anderson, Wealleans, and Ray, 2018]. Очень важно, чтобы клиницисты и технические специалисты были знакомы с преимуществами и недостатками автоматизированного производства, поскольку эти процедуры продолжают развиваться и становятся неотъемлемой частью стоматологии [Abduo, Lyons, and Bennamoun, 2014].

Особый интерес вызывает точность 3D-печати, что подтверждается ростом числа публикаций на эту тему. Точность технологии 3D-печати имеет большое значение для клинических применений [Wang et al., 2015; Ishida and Miyasaka, 2016; Jeong, Lee, and Lee 2018; Kalman 2018]. Компьютеры теперь используются для создания точно детализиро- 
ванных моделей, которые могут быть оценены с разных точек зрения в процессе, известном как автоматизированное проектирование (САПР). Для материализации виртуальных объектов с помощью САПР был разработан процесс автоматизированного производства (CAM). Чтобы преобразовать виртуальный файл в реальный объект, САМ работает с помощью машины, подключенной к компьютеру, аналогично принтеру или периферийному устройству [Nayar, Bhuminathan, and Bhat, 2015]. Производство CAD/CAM является более точным, чем технология использования литьевых восков, так как она основана на минимальном вмешательстве человека [Зотова, Вдовенко, 2015]. Автоматизированные производственные процедуры, несомненно, изменят многие аспекты стоматологии в будущем, особенно в отношении простоты лечения и времени производства [Salmi et al., 2013; Дьяченко и др., 2015]. Также абатменты и каркасы имплантатов CAD/CAM лучше подходят, чем обычные литые компоненты. Спроектированная поверхность имплантата имеет преимущество в плавной обработке с определенными характеристиками, что облегчает запись точной геометрии с минимальными неровностями [Abduo and Lyons, 2013].

На сегодняшний день наличие трехмерных (3D) настольных принтеров позволяет экономически эффективно производить направляющие для свёрл в зуботехнических лабораториях, что способствует широкому внедрению данной технологии в имплантологию [Chen et al., 2014; Лазаренко и др., 2018; Kalman, 2018]. Современные исследования показывают высокую точность изготовленных направляющих, что устраняет внешние факторы, существующие в рабочем процессе управляемой хирургии [Neumeister, Schulz, and Glodecki, 2017]. Кроме того, 3D-печать стала неотъемлемой частью и ортопедической стоматологии благодаря своей точности [Шустова, Шустов, 2016]. Использование метода фрезерования с использованием системы CAD/CAM и метода аддитивного производства 3D-печати для изготовления зубных протезов неуклонно развивается, привлекая большой интерес в области стоматологии [Аствацатрян, Гажва, 2017; Jeong, Lee, and Lee, 2018]. Зубной оттиск - это негативный отпечаток структуры полости рта, используемый для изготовления зубного протеза или реставрации [Митин и др., 2019]. Точное измерение имеет решающее значение для изготовления зубных реставраций с адекватной подгонкой. Несоответствие имплантата протезу по неточному вычислению приводит к механическим и биологическим осложнениям [Rhee et al., 2015]. В прошлом, используя традиционный метод, оттиск получали путем заливки полужесткого материала в стоматологический оттискный лоток, который затем затвердевал. Эта процедура была неудобна для пациентов, и точность измерений существенно зависела от уровня мастерства и техники практикующих. Однако прогресс цифровых технологий и внедрение автоматизированного проектирования / автоматизированного производства (CAD/CAM) внесли большие изменения в традиционный способ производства, повышая точность и качество предоставляемых услуг.

Будущие направления исследований должны включать оценку клинических результатов лечения с использованием 3D-печатных объектов [Anderson, Wealleans and Ray, 2018]. Исследование в этой области проводили Zhang H.R.,Yin L.F., Liu Y.L., Yan L.Y., Wang N., Liu G., An X.L., Liu B. [Zhang et al., 2018]. Они построили цифровые стоматологические модели с помощью конусно-лучевой компьютерной томографии (СВСТ), создали виртуальную модель с помощью 3D-печати и определили точности 3D-печати стоматологической модели путем сравнения результата с традиционным стоматологическим слепком. Авторы сделали следующее заключение: модели 3D-печати имели более высокую точность по сравнению с традиционными литыми моделями. Модели 3D-печати FDM (моделирование плавленого осаждения) показывают высокую степень точности. Таким образом, эти модели подходят для клинической практики. Сегодня CAD/CAM является единственным средством производства прочных, безметалловых, имитирующих цвет зуба компонентов в стоматологической практике, включая имплантологию, а также предоставляет возможность изготовления непрямых реставраций [Miyazaki and Hotta, 2011; Abduo and Lyons, 2013]. Метод CAD/CAM использует современные технологии. Сканер оцифро- 
вывает подготовленный зуб, и затем каркас или реставрация изготавливается в соответствии с ранее установленной конструкцией. Кроме того, системы CAD/CAM были разработаны для устранения или минимизации потенциальных источников ошибок, присутствующих в традиционных технологиях производства [Martins et al., 2012].

Однако точность 3D-печати зависит от ряда критериев. Так, исследования, проведенные Tahayeri A., Morgan M., Fugolin A.P., Bompolaki D., Athirasala A., Pfeifer C.S., Ferracane J.L., Bertassoni L.E. [Tahayeri et al., 2018], доказывают, что ориентация печати и настройка цвета смолы оказывают влияние на точность печати. Установка цвета смолы и толщина слоя печати влияют на интенсивность лазерного луча. Авторы также обнаружили, что толщина слоя 3D-печати не оказывает существенного влияния на механические свойства 3D-печатных смол. Для повышения точности 3D-печати стоматологических материалов в будущем следует проводить работы с использованием систем 3D-печати, позволяющих оптимизировать параметры печати в зависимости от выбранной смолы. Кроме того, в зависимости от метода 3D-печати машины и внешних факторов точность варьируется, даже если печатается одна и та же модель автоматизированного проектирования (САПР) [Tahayeri et al., 2018, Kim et al., 2019]. Существует ряд доступных 3D-принтеров с возможностью печати различных объектов с использованием разнообразных технологий печати. Наиболее часто используемые 3D-принтеры представляют собой твердотельные устройства моделирования плавленого осаждения (FDM), в которых тонкая пластиковая нить используется для укладки слоев для создания пластикового объекта [Милев и др., 2017]. Порошковые 3D-принтеры, например, селективное лазерное спекание, используют нейлон или аналогичный термопластичный порошок, который локально расплавляется лазерным лучом. В последнее время были внедрены различные технологии 3D-печати на жидкой основе, такие как стереолитографический аппарат (SLA) и цифровая обработка света (DLP), а также PolyJet (фотополимерный струйный). В этих технологиях ультрафиолетовая (УФ) отверждаемая смола полимеризуется с образованием желаемой формы источниками света [Lee et al., 2015].

Kim T., Lee S., Kim G.B., Hong D., Kwon J., Park J.W., Kim N. [Kim et al., 2019] проводили исследование, в котором оценивали различия между моделью САПР и печатными частями с упрощенной направляющей, разработанной на основе направляющей имплантата, и сравнивали точность между тремя типами 3D-принтеров. Направляющая имплантата верхней и нижней челюстей, выполненная из сложных анатомических структур, трудно поддается точному измерению. Для точных измерений были разработаны 16 упрощенных направляющих, основанных на направляющих имплантатов верхней и нижней челюстей, которые были изготовлены с использованием следующих трех различных технологий 3Dпринтера: фотополимерный струйный (PolyJet), стереолитографический аппарат (SLA) и струйная печать (MJP). Каждая упрощенная направляющая измерялась 4 раза цифровыми штангенциркулями для 20 линейных измерений. Измеренные упрощенные направляющие сравнивались с моделью САПР, а также сравнивалась точность 3D-принтеров. Исследователи пришли к следующему выводу: при сравнении моделей CAПР и 3D-печатных частей упрощенных направляющих имплантатов наблюдались значительные различия в точности. 3D-принтеры PolyJet и SLA соответствовали требуемой точности для клинических применений в стоматологии. Однако наиболее подходящий 3D-принтер должен быть выбран с учетом всех обстоятельств конкретной клинической ситуации (состояние пациента, оборудование клиники, навыки врача и т. д.).

Таким образом, 3D-печать обладает рядом преимуществ, которые позволяют ей конкурировать с традиционными методами изготовления моделей и даже вытеснять их. К таковым преимуществам относится высокая точность, что является ключевым фактором в эффективности проводимого лечения и позволяет повсеместно использовать данные модели в клинической практике; сокращение времени операции; высокие эстетические качества; перспектива развития персонализированной медицины, что является ключевой целью современной медицины; простота использования и возможность создания объектов 
различной сложности. Все это способствует использованию данной технологии в диагностике и лечении стоматологических заболеваний всех направленностей. Перечисленные преимущества стимулируют создание новых и совершенствование старых моделей 3Dпринтеров и программ печати. Следовательно, внедрение трехмерной печати в стоматологию позволяет улучшить качество оказываемой пациенту медицинской помощи.

\section{Список литературы}

1. Аствацатрян Л.Э., Гажва С.И. 2017. Современные аспекты использования 3Dтехнологий в изготовлении съемных зубных протезов. Современные проблемы науки и образования, (5): 194.

2. Визер Ю.Ю., Елали А.Х. 2017. Технологии 3D-печати в медицине и стоматологии. Естественнонаучные основы медико-биологических знаний, с. 114-116.

3. Донских Д.А. 2017. Использование 3D-принтера в стоматологии. Бюллетень медицинских Интернет-конференций, 7 (1): 401.

4. Дьяченко Д.Ю., Гаврикова С.В., Михальченко Д.В.,. Михальченко А.В. 2015. Применение 3D-печати в стоматологии для изготовления провизорных ортопедических конструкций. Электронный научно-образовательный вестник здоровье и образование в XXI веке, 17 (3): $5-7$.

5. Зотова А.А., Вдовенко К.Д. 2015. Актуальность применения 3D-принтеров в современной стоматологии. Бюллетень медицинских Интернет-конференций, 5 (11): 1284.

6. Коровкина В.С. 2018. Применение 3D-печати и 3D-сканирования в стоматологии. Физика и медицина: создавая будущее, с. 256-258.

7. Лазаренко В.А., Иванов С.В., Иванов И.С., Объедков Е.Г., Беликов Л.Н., Объедкова Н.Ю., Денисенко А.И. 2018. Использование 3D-принтеров в хирургии (обзор литературы). Курский научно-практический вестник «Человек и его здоровье», (4): 61-65.

8. Милев Минко Милчев, Симов Максим Иванов, Ангелова Светлана Пенева, Великова Валентина Степанова 2017. Применение 3D-технологий в эстетической дентальной медицине. Научные исследования: векторы развития, с. 50-53.

9. Митин Н.Е., Захарова И.В., Перминов Е.С., Калиновский С.И. Исследование влияния иммедиат-протезов с амортизирующей промежуточной частью на репарацию костной ткани в постэкстракционный период и остеоинтеграцию имплантатов в области резцов верхней челюсти. Клиническая стоматология. 2019. 2 (90): 80-82.

10. Чевычелова О.Н., Зубкова А.А. 2019. Реализация 3D-сканирования и 3D-печати в ортопедической стоматологии. Приемущества и недостатки. World Science: Problems and Innovations, c. 239-241.

11. Шустова В.А., Шустов М.А. 2016. Применение 3D-технологий в ортопедической стоматологии. Санкт-Петербург, Общество с ограниченной ответственностью «Издательство «СпецЛит», с. 159.

12. Abduo Jaafar and Karl Lyons. 2013. Rationale for the Use of CAD/CAM Technology in Implant Prosthodontics. International Journal of Dentistry, p. 1-8.

13. Abduo Jaafar, Karl Lyons and Mohammed Bennamoun. 2014. Trends in Computer-Aided Manufacturing in Prosthodontics: A Review of the Available Streams. International Journal of Dentistry p. $1-15$.

14. Anderson J., Wealleans J. and Ray J. 2018. Endodontic Applications of 3D Printing. International Endodontic Journal. Blackwell Publishing Ltd 51(9): 1005-1018.

15. Brown Gregory B., G. Fräns Currier, Onur Kadioglu and J. Peter Kierl. 2018. Accuracy of 3-Dimensional Printed Dental Models Reconstructed from Digital Intraoral Impressions. American Journal of Orthodontics and Dentofacial Orthopedics 154 (5): 733-39.

16. Chan Hsun Liang, Kelly Misch and Hom Lay Wang. 2010. Dental Imaging in Implant Treatment Planning. Implant Dentistry 19 (4): 288-98.

17. Chen Jianyu, Zhiguang Zhang, Xianshuai Chen, Chunyu Zhang, Gong Zhang and Zhewu Xu. 2014. Design and Manufacture of Customized Dental Implants by Using Reverse Engineering and Selective Laser Melting Technology. Journal of Prosthetic Dentistry 112 (5): 1088-1095. 
18. Choi Jong Woo and Namkug Kim. 2015. Clinical Application of Three-Dimensional Printing Technology in Craniofacial Plastic Surgery. Archives of Plastic Surgery. Korean Society of Plastic and Reconstructive Surgeons 42(3): 267-277.

19. Dawood Andrew, Susan Tanner and Iain Hutchison. 2013. Computer Guided Surgery for Implant Placement and Dental Rehabilitation in a Patient Undergoing Sub-Total Mandibulectomy and Microvascular Free Flap Reconstruction. Journal of Oral Implantology 39 (4): 497-502.

20. Dodziuk Helena. 2016. Applications of 3D Printing in Healthcare. Kardiochirurgia i Torakochirurgia Polska. Termedia Publishing House Ltd 13(3): 283-293.

21. Ishida Yoshiki and Taira Miyasaka. 2016. Dimensional Accuracy of Dental Casting Patterns Created by 3D Printers. Dental Materials Journal 35 (2): 250-56.

22. Jeong Yoo Geum, Wan Sun Lee and Kyu Bok Lee. 2018. Accuracy Evaluation of Dental Models Manufactured by CAD/CAM Milling Method and 3D Printing Method. Journal of Advanced Prosthodontics 10 (3): 245-51.

23. Kalman Les. 2018. 3D Printing of a Novel Dental Implant Abutment. Journal of Dental Research, Dental Clinics, Dental Prospects 12 (4): 299-303.

24. Kasparova Magdalena, Lucie Grafova, Petr Dvorak, Tatjana Dostalova, Ales Prochazka, Hana Eliasova, Josef Prusa and Soroush Kakawand. 2013. Possibility of Reconstruction of Dental Plaster Cast from 3D Digital Study Models. BioMedical Engineering Online 12 (1).

25. Kim Taehun, Sangwook Lee, Bae Kim, Dayeong Hong, Jinhee Kwon, Jae-woo Park and Namkug Kim. 2019. Accuracy of a Simplified 3D Printed Implant Surgical Guide. The Journal of Prosthetic Dentistry S0022-3913(19)30416-0.

26. Lee Keun-Young, Jin-Woo Cho, Na-Young Chang, Jong-Moon Chae, Kyung-Hwa Kang, Sang-Cheol Kim and Jin-Hyoung Cho. 2015. Accuracy of Three-Dimensional Printing for Manufacturing Replica Teeth. The Korean Journal of Orthodontics 45 (5): 217.

27. Liu Qingbin, Ming C. Leu and Stephen M. Schmitt. 2006. Rapid Prototyping in Dentistry: Technology and Application. The International Journal of Advanced Manufacturing Technology 29 (3-4): 317-35.

28. Louvrier A., P. Marty, A. Barrabé, E. Euvrard, B. Chatelain, E. Weber, and C. Meyer. 2017. How Useful Is 3D Printing in Maxillofacial Surgery? Journal of Stomatology, Oral and Maxillofacial Surgery. Elsevier Masson SAS 118(4): 206-212.

29. Martins Leandro Moura, Fabio Cesar Lorenzoni, Alcides Oliveira de Melo, Luciana Mendonça da Silva, José Luiz G. de Oliveira, Pedro Cesar Garcia de Oliveira and Gerson Bonfante. 2012. Internal Fit of Two All-Ceramic Systems and Metal-Ceramic Crowns. Journal of Applied Oral Science 20 (2): 235-40.

30. Miyazaki T. and Y. Hotta. 2011. CAD/CAM Systems Available for the Fabrication of Crown and Bridge Restorations. Australian Dental Journal 56 (SUPPL. 1): 97-106.

31. Moser Norman, Petra Santander and Anja Quast. 2018. From 3D Imaging to 3D Printing in Dentistry - a Practical Guide. International Journal of Computerized Dentistry 21 (4): 345-56.

32. Nayar Sanjna, S. Bhuminathan and Wasim Bhat. 2015. Rapid Prototyping and Stereolithography in Dentistry. Journal of Pharmacy and Bioallied Sciences. Medknow Publications 7(1): 216-219.

33. Neumeister André, Linda Schulz and Christoph Glodecki. 2017. Investigations on the Accuracy of 3D Printed Drill Guides for Dental Implantology. International Journal of Computerized Dentistry 20 (1): 35-51.

34. Oberoi Gunpreet, Sophie Nitsch, Michael Edelmayer, Klara Janjic, Anna Sonja Müller and Hermann Agis. 2018. 3D Printing-Encompassing the Facets of Dentistry. Frontiers in Bioengineering and Biotechnology. Frontiers Media S.A. 6: 172.

35. Rhee Ye Kyu, Yoon Hyuk Huh, Lee Ra Cho and Chan Jin Park. 2015. Comparison of Intraoral Scanning and Conventional Impression Techniques Using 3-Dimensional Superimposition. Journal of Advanced Prosthodontics 7 (6): 460-67.

36. Salmi Mika, Kaija Stiina Paloheimo, Jukka Tuomi, Jan Wolff and Antti Mäkitie. 2013. Accuracy of Medical Models Made by Additive Manufacturing (Rapid Manufacturing). Journal of Cranio-Maxillofacial Surgery 41 (7): 603-9.

37. Shah Pratik and B.S. Chong. 2018. 3D Imaging, 3D Printing and 3D Virtual Planning in Endodontics. Clinical Oral Investigations. Springer Verlag 22(2): 641-654. 
38. Sinn Douglas P., Joseph E. Cillo and Brett A. Miles. 2006. Stereolithography for Craniofacial Surgery. Journal of Craniofacial Surgery 17 (5): 869-75.

39. Tahayeri Anthony, Mary Catherine Morgan, Ana P. Fugolin, Despoina Bompolaki, Avathamsa Athirasala, Carmem S. Pfeifer, Jack L. Ferracane and Luiz E. Bertassoni. 2018. 3D Printed versus Conventionally Cured Provisional Crown and Bridge Dental Materials. Dental Materials 34 (2): 192-200.

40. Wang Chen and Lin. 2019. A Collaborative and Ubiquitous System for Fabricating Dental Parts Using 3D Printing Technologies. Healthcare 7 (3): 103.

41. Wang Ning, Jie Li, Xiaolong Wang, Gang Liu and Bin Liu. 2015. 3D Printing Personalized Implant Manufactured via Fused Deposition Modeling: An Accuracy Research. Hua Xi Kou Qiang Yi Xue Za Zhi. West China Journal of Stomatology 33 (5): 509-12.

42. Zhang Hui Rong, Le Feng Yin, Yan Li Liu, Li Yi Yan, Ning Wang, Gang Liu, Xiao Li An and Bin Liu. 2018. Fabrication and Accuracy Research on 3D Printing Dental Model Based on Cone Beam Computed Tomography Digital Modeling. Hua Xi Kou Qiang Yi Xue Za Zhi. West China Journal of Stomatology 36 (2): 156-61.

\section{References}

1. Astvacatrjan L.Je., Gazhva S.I. 2017. Sovremennye aspekty ispol'zovanija 3D-tehnologij v izgotovlenii s"emnyh zubnyh protezov [Modern aspects of using 3D technologies in the manufacture of removable dentures]. Sovremennye problemy nauki i obrazovanija, (5): 194.

2. Vizer Ju.Ju., Elali A.H. 2017. Tehnologii 3D-pechati v medicine i stomatologii [3D printing technologies in medicine and dentistry]. Estestvennonauchnye osnovy mediko-biologicheskih znanij, s. $114-116$.

3. Donskih D.A. 2017. Ispol'zovanie 3D-printera v stomatologii [Using a 3D printer in dentistry]. Bjulleten' medicinskih Internet-konferencij, 7 (1): 401.

4. D'jachenko D.Ju., Gavrikova S.V., Mihal'chenko D.V., Mihal'chenko A.V. 2015. Primenenie 3D-pechati v stomatologii dlja izgotovlenija provizornyh ortopedicheskih konstrukcij [The use of 3D printing in dentistry for the manufacture of orthopedic structures]. Jelektronnyj nauchno-obrazovatel'nyj vestnik zdorov'e i obrazovanie v XXI veke, 17 (3): 5-7.

5. Zotova A.A., Vdovenko K.D. 2015. Aktual'nost' primenenija 3D-printerov v sovremennoj stomatologii [Relevance of 3D printers in modern dentistry]. Bjulleten' medicinskih Internet-konferencij, 5 (11): 1284.

6. Korovkina V.S. 2018. Primenenie 3D-pechati i 3D-skanirovanija v stomatologii [Application of 3D printing and 3D scanning in dentistry]. Fizika i medicina: sozdavaja budushhee, s. 256-258.

7. Lazarenko V.A., Ivanov S.V., Ivanov I.S., Ob"edkov E.G., Belikov L.N., Ob"edkova N. Ju., Denisenko A.I. 2018. Ispol'zovanie 3D-printerov v hirurgii (obzor literatury) [Using 3D printers in surgery (literature review)]. Kurskij nauchno-prakticheskij vestnik «Chelovek i ego zdorov'e», (4): 61-65.

8. Milev Minko Milchev, Simov Maksim Ivanov, Angelova Svetlana Peneva, Velikova Valentina Stepanova 2017. Primenenie 3D-tehnologij v jesteticheskoj dental'noj medicine [Application of 3D technologies in aesthetic dental medicine]. Nauchnye issledovanija: vektory razvitija, s. 50-53.

9. Mitin N.E., Zaharova I.V., Perminov E.S., Kalinovskij S.I. Issledovanie vlijanija immediatprotezov s amortizirujushhej promezhutochnoj chast'ju na reparaciju kostnoj tkani v postjekstrakcionnyj period i osteointegraciju implantatov v oblasti rezcov verhnej cheljusti [Investigation of the effect of immediate prostheses with a cushioning intermediate part on bone repair in the post-extraction period and osteointegration of implants in the upper jaw incisors]. Klinicheskaja stomatologija. 2019. 2 (90): 80-82.

10. Chevychelova O.N., Zubkova A.A. 2019. Realizacija 3D-skanirovanija i 3D-pechati v ortopedicheskoj stomatologii. Priemushhestva i nedostatki [Implementation of 3D scanning and 3D printing in orthopedic dentistry. Advantages and disadvantages]. World Science: Problems and Innovations, s. 239-241.

11. Shustova V.A., Shustov M.A. 2016. Primenenie 3D-tehnologij v ortopedicheskoj stomatologii [Application of 3D technologies in orthopedic dentistry]. Sankt-Peterburg, Obshhestvo s ogranichennoj otvetstvennost'ju «Izdatel'stvo «SpecLit», s. 159.

12. Abduo Jaafar and Karl Lyons. 2013. Rationale for the Use of CAD/CAM Technology in Implant Prosthodontics. International Journal of Dentistry, p. 1-8. 
13. Abduo Jaafar, Karl Lyons and Mohammed Bennamoun. 2014. Trends in Computer-Aided Manufacturing in Prosthodontics: A Review of the Available Streams. International Journal of Dentistry p. $1-15$.

14. Anderson J., Wealleans J. and Ray J. 2018. Endodontic Applications of 3D Printing. International Endodontic Journal. Blackwell Publishing Ltd 51(9): 1005-1018.

15. Brown Gregory B., G. Fräns Currier, Onur Kadioglu and J. Peter Kierl. 2018. Accuracy of 3-Dimensional Printed Dental Models Reconstructed from Digital Intraoral Impressions. American Journal of Orthodontics and Dentofacial Orthopedics 154 (5): 733-39.

16. Chan Hsun Liang, Kelly Misch and Hom Lay Wang. 2010. Dental Imaging in Implant Treatment Planning. Implant Dentistry 19 (4): 288-98.

17. Chen Jianyu, Zhiguang Zhang, Xianshuai Chen, Chunyu Zhang, Gong Zhang and Zhewu Xu. 2014. Design and Manufacture of Customized Dental Implants by Using Reverse Engineering and Selective Laser Melting Technology. Journal of Prosthetic Dentistry 112 (5): 1088-1095.

18. Choi Jong Woo and Namkug Kim. 2015. Clinical Application of Three-Dimensional Printing Technology in Craniofacial Plastic Surgery. Archives of Plastic Surgery. Korean Society of Plastic and Reconstructive Surgeons 42(3): 267-277.

19. Dawood Andrew, Susan Tanner and Iain Hutchison. 2013. Computer Guided Surgery for Implant Placement and Dental Rehabilitation in a Patient Undergoing Sub-Total Mandibulectomy and Microvascular Free Flap Reconstruction. Journal of Oral Implantology 39 (4): 497-502.

20. Dodziuk Helena. 2016. Applications of 3D Printing in Healthcare. Kardiochirurgia i Torakochirurgia Polska. Termedia Publishing House Ltd 13(3): 283-293.

21. Ishida Yoshiki and Taira Miyasaka. 2016. Dimensional Accuracy of Dental Casting Patterns Created by 3D Printers. Dental Materials Journal 35 (2): 250-56.

22. Jeong Yoo Geum, Wan Sun Lee and Kyu Bok Lee. 2018. Accuracy Evaluation of Dental Models Manufactured by CAD/CAM Milling Method and 3D Printing Method. Journal of Advanced Prosthodontics 10 (3): 245-51.

23. Kalman Les. 2018. 3D Printing of a Novel Dental Implant Abutment. Journal of Dental Research, Dental Clinics, Dental Prospects 12 (4): 299-303.

24. Kasparova Magdalena, Lucie Grafova, Petr Dvorak, Tatjana Dostalova, Ales Prochazka, Hana Eliasova, Josef Prusa and Soroush Kakawand. 2013. Possibility of Reconstruction of Dental Plaster Cast from 3D Digital Study Models. BioMedical Engineering Online 12 (1).

25. Kim Taehun, Sangwook Lee, Bae Kim, Dayeong Hong, Jinhee Kwon, Jae-woo Park and Namkug Kim. 2019. Accuracy of a Simplified 3D Printed Implant Surgical Guide. The Journal of Prosthetic Dentistry S0022-3913(19)30416-0.

26. Lee Keun-Young, Jin-Woo Cho, Na-Young Chang, Jong-Moon Chae, Kyung-Hwa Kang, Sang-Cheol Kim and Jin-Hyoung Cho. 2015. Accuracy of Three-Dimensional Printing for Manufacturing Replica Teeth. The Korean Journal of Orthodontics 45 (5): 217.

27. Liu Qingbin, Ming C. Leu and Stephen M. Schmitt. 2006. Rapid Prototyping in Dentistry: Technology and Application. The International Journal of Advanced Manufacturing Technology 29 (3-4): 317-35.

28. Louvrier A., P. Marty, A. Barrabé, E. Euvrard, B. Chatelain, E. Weber, and C. Meyer. 2017. How Useful Is 3D Printing in Maxillofacial Surgery? Journal of Stomatology, Oral and Maxillofacial Surgery. Elsevier Masson SAS 118(4): 206-212.

29. Martins Leandro Moura, Fabio Cesar Lorenzoni, Alcides Oliveira de Melo, Luciana Mendonça da Silva, José Luiz G. de Oliveira, Pedro Cesar Garcia de Oliveira and Gerson Bonfante. 2012. Internal Fit of Two All-Ceramic Systems and Metal-Ceramic Crowns. Journal of Applied Oral Science 20 (2): $235-40$.

30. Miyazaki T. and Y. Hotta. 2011. CAD/CAM Systems Available for the Fabrication of Crown and Bridge Restorations. Australian Dental Journal 56 (SUPPL. 1): 97-106.

31. Moser Norman, Petra Santander and Anja Quast. 2018. From 3D Imaging to 3D Printing in Dentistry - a Practical Guide. International Journal of Computerized Dentistry 21 (4): 345-56.

32. Nayar Sanjna, S. Bhuminathan and Wasim Bhat. 2015. Rapid Prototyping and Stereolithography in Dentistry. Journal of Pharmacy and Bioallied Sciences. Medknow Publications 7(1): 216-219. 
33. Neumeister André, Linda Schulz and Christoph Glodecki. 2017. Investigations on the Accuracy of 3D Printed Drill Guides for Dental Implantology. International Journal of Computerized Dentistry 20 (1): 35-51.

34. Oberoi Gunpreet, Sophie Nitsch, Michael Edelmayer, Klara Janjic, Anna Sonja Müller and Hermann Agis. 2018. 3D Printing-Encompassing the Facets of Dentistry. Frontiers in Bioengineering and Biotechnology. Frontiers Media S.A. 6: 172.

35. Rhee Ye Kyu, Yoon Hyuk Huh, Lee Ra Cho and Chan Jin Park. 2015. Comparison of Intraoral Scanning and Conventional Impression Techniques Using 3-Dimensional Superimposition. Journal of Advanced Prosthodontics 7 (6): 460-67.

36. Salmi Mika, Kaija Stiina Paloheimo, Jukka Tuomi, Jan Wolff and Antti Mäkitie. 2013. Accuracy of Medical Models Made by Additive Manufacturing (Rapid Manufacturing). Journal of Cranio-Maxillofacial Surgery 41 (7): 603-9.

37. Shah Pratik and B.S. Chong. 2018. 3D Imaging, 3D Printing and 3D Virtual Planning in Endodontics. Clinical Oral Investigations. Springer Verlag 22(2): 641-654.

38. Sinn Douglas P., Joseph E. Cillo and Brett A. Miles. 2006. Stereolithography for Craniofacial Surgery. Journal of Craniofacial Surgery 17 (5): 869-75.

39. Tahayeri Anthony, Mary Catherine Morgan, Ana P. Fugolin, Despoina Bompolaki, Avathamsa Athirasala, Carmem S. Pfeifer, Jack L. Ferracane and Luiz E. Bertassoni. 2018. 3D Printed versus Conventionally Cured Provisional Crown and Bridge Dental Materials. Dental Materials 34 (2): 192-200.

40. Wang Chen and Lin. 2019. A Collaborative and Ubiquitous System for Fabricating Dental Parts Using 3D Printing Technologies. Healthcare 7 (3): 103.

41. Wang Ning, Jie Li, Xiaolong Wang, Gang Liu and Bin Liu. 2015. 3D Printing Personalized Implant Manufactured via Fused Deposition Modeling: An Accuracy Research. Hua Xi Kou Qiang Yi Xue Za Zhi. West China Journal of Stomatology 33 (5): 509-12.

42. Zhang Hui Rong, Le Feng Yin, Yan Li Liu, Li Yi Yan, Ning Wang, Gang Liu, Xiao Li An and Bin Liu. 2018. Fabrication and Accuracy Research on 3D Printing Dental Model Based on Cone Beam Computed Tomography Digital Modeling. Hua Xi Kou Qiang Yi Xue Za Zhi. West China Journal of Stomatology 36 (2): 156-61.

\section{Ссылка для цитирования статьи For citation}

Иванова В.А., Борисов В.В., Платонова В.В., Даньшина С.Д. 2020. Высокая точность конструкций при применении 3D-печати в имплантологии (обзор литературы). Актуальные проблемы медицины, 43(1): 93-101. DOI

Ivanova V.A., Borisov V.V., Platonova V.V., Danshina S.D. 2020. High accuracy of designs when using 3D printing in implantology (review of literature). Challenges in Modern Medicine, 43(1): 93-101 (in Russian). DOI 